\title{
A patient on RIPE therapy presenting with recurrent isoniazid-associated pleural effusions: a case report
}

\author{
Vanja Varenika and Paul D Blanc ${ }^{*}$
}

\begin{abstract}
Introduction: The clinical scenario of a new or worsening pleural effusion following the initiation of antituberculous therapy has been classically referred to as a 'paradoxical' pleural response, presumably explained by an immunological rebound phenomenon. Emerging evidence suggests that there also may be a role for a lupusrelated reaction in the pathophysiology of this disorder.

Case presentation: An 84-year-old Asian man treated with isoniazid, along with rifampin, pyrazinamide and ethambutol for suspected extrapulmonary tuberculosis, presented with a recurrent pleural effusion, his third episode since the initiation of this therapy. The first effusion occurred one month after the start of treatment, without any prior evidence of pulmonary tuberculosis involvement. Follow-up testing, including thoracoscopic pleural biopsies, never confirmed tuberculosis infection. Further evaluation yielded serological evidence suggesting drug-induced lupus. No effusions recurred following the discontinuation of isoniazid, although other antituberculosis medications were continued.
\end{abstract}

Conclusion: The immunological rebound construct is inconsistent with the evolution of this case, which indicates rather that drug-induced lupus may explain at least some cases of new pleural effusions following the initiation of isoniazid.

\section{Introduction}

Pleural effusions are frequently observed in pulmonary Mycobacterium tuberculosis infection (TB), occurring in up to $30 \%$ of cases [1]. New onset pleural effusions following weeks of antituberculous treatment are far less common. This clinical scenario, of a delayed emerging effusion following the initiation of therapy, has been referred to as a 'paradoxical' response [2,3]. Published reports often attribute this to an immunological rebound, in which an excessive antigen load from bacteriolysis is presumed to interact with a 'revved-up' cell-mediated immunity. This model of a pleural-based response draws on immunological phenomena related to tuberculous lymphadenitis and intracranial tuberculoma [4]. While this has been the traditional explanation, cases have emerged that suggest a role for drug-induced lupus mediating this phenomenon

\footnotetext{
* Correspondence: Paul.Blanc@ucsf.edu Department of Occupational and Environmental Medicine, University of California, San Francisco, 350 Parnassus Avenue, Room 609, San Francisco, CA 94143-0924, USA
}

(c) 2011 Varenika and Blanc; licensee BioMed Central Ltd. This is an Open Access article distributed under the terms of the Creative Commons Attribution License (http://creativecommons.org/licenses/by/2.0), which permits unrestricted use, distribution, and reproduction in any medium, provided the original work is properly cited.

\section{Case presentation}

An 84-year-old Asian man was admitted to hospital for dyspnea and was found to have a large, recurrent rightsided pleural effusion. This was the third time our patient presented with an effusion since the initiation of rifampin, isoniazid, pyrazinamide and ethambutol (RIPE) therapy for presumed extrapulmonary TB. Nine months prior to this presentation, our patient underwent a screening colonoscopy with a biopsy that reportedly yielded a single granulomatous, acid-fast stain-positive, but TB culture-negative lesion. No anti-TB treatment was initiated at that time. The purportedly acid-fast stain-positive specimen could 
not be located subsequently for re-review. A repeat colonoscopy with biopsy at six months demonstrated no granulomas or other evidence of TB. There was no radiographic evidence of pulmonary $\mathrm{TB}$ and interval sputum cultures were negative, as was a serum QuantiFERON-TB Gold test. Nevertheless, our patient was started on RIPE combination therapy.

Four weeks after the initiation of this regimen (two months prior to the episode described in this report), our patient complained of dyspnea, cough and weakness. He was admitted to hospital with the initial right-sided pleural effusion presentation (Figure 1). Thoracocentesis yielded exudative, culture- and stain-negative pleural fluid (Table 1). Our patient was given a presumptive diagnosis of a paradoxical tuberculous effusion and discharged on a tapering course of systemic glucocorticosteroids while he continued on RIPE therapy. Two months later, he was readmitted with a similar clinical picture. A further liter of exudative fluid was removed by repeat thoracocentesis (Figure 1 and Table 1).
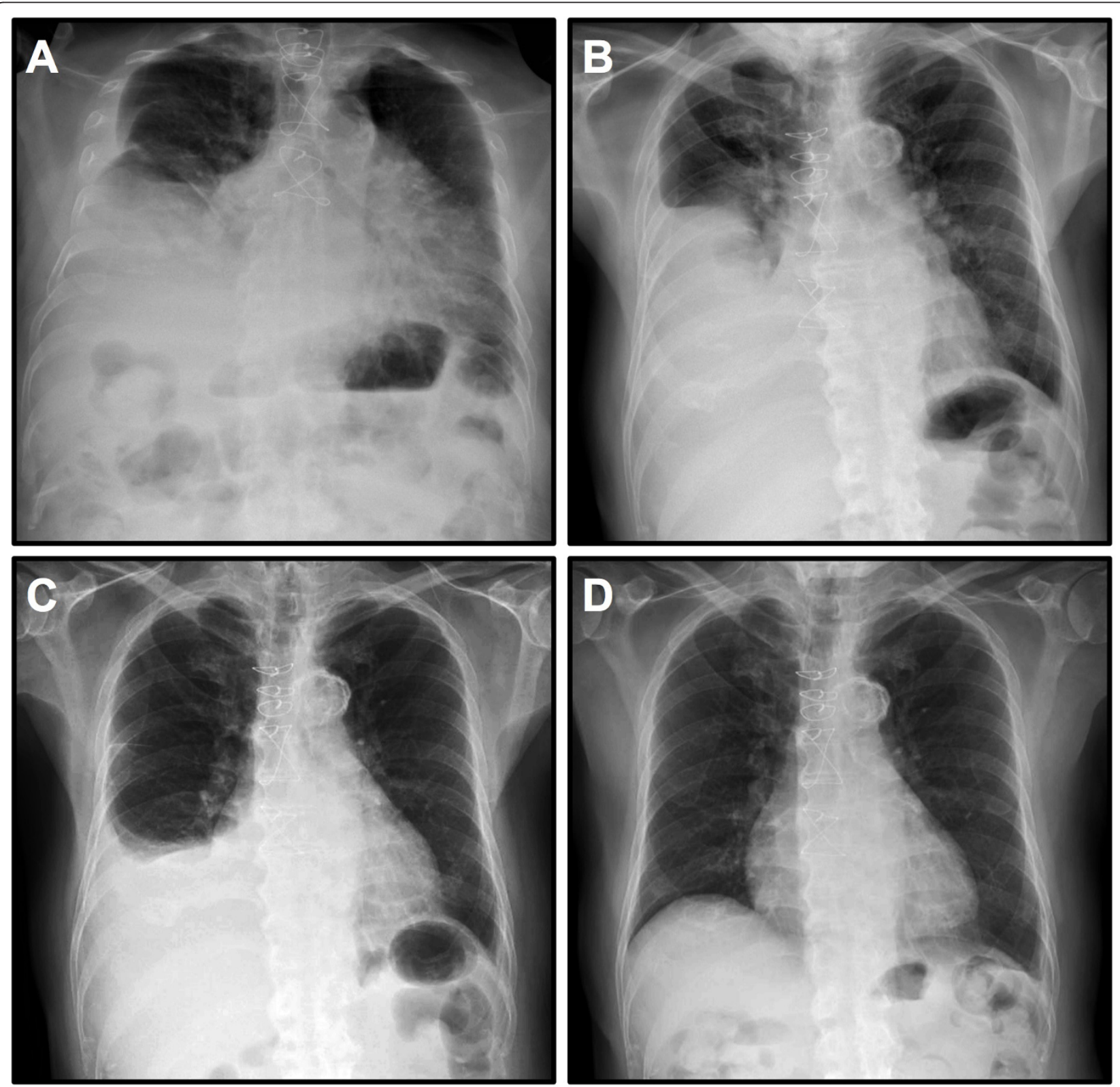

Figure 1 Chest X-ray images. (A) Large pleural effusion in his right lung field found on the first admission. (B) Large pleural effusion in his right lung field on the second admission. (C) Pleural effusion in his right lung field on the current admission, two weeks after the last previous thoracocentesis. (D) Clear chest X-ray six months after the discontinuation of isoniazid. 
Table 1 Laboratory values for the pleural fluid collected from the three effusions our patient manifested.

\begin{tabular}{lccc}
\hline & $\begin{array}{c}\text { 1st } \\
\text { Effusion }\end{array}$ & $\begin{array}{c}\text { 2nd } \\
\text { Effusion }\end{array}$ & $\begin{array}{c}\text { 3rd } \\
\text { Effusion }\end{array}$ \\
\hline Color & Red & Red & Yellow \\
Red blood cells $(\times \mathbf{1 0}$ /L) & 57.25 & 107 & 5.575 \\
White blood cells ( $\times \mathbf{1 0}$ /L) & 2.075 & 0.219 & 0.17 \\
Neutrophils & $11 \%$ & $1 \%$ & $1 \%$ \\
Lymphocytes & $78 \%$ & $41 \%$ & $96 \%$ \\
Monocytes & $11 \%$ & $55 \%$ & $2 \%$ \\
Eosinophils & $0 \%$ & $3 \%$ & $1 \%$ \\
pH & 7.81 & - & - \\
Albumin (g/dL) & 2.1 & - & - \\
Amylase (U/L) & - & - & 67 \\
Glucose (mg/dL) & 257 & 212 & 125 \\
Protein (g/dL) & 4.2 & 3.3 & 3.3 \\
Lactate dehydrogenase (IU/L) & 104 & 200 & 224 \\
Triglyceride (mg/dL) & - & - & 21 \\
Complement (CH50) & - & - & 10 \\
Adenosine deaminase (U/L) & - & - & 23.8 \\
ANA Titer & - & - & $<1: 40$ \\
Serum lactate dehydrogenase & 159 & - & - \\
(IU/L) & & & - \\
Serum Protein (g/dL) & 6.2 & - & \\
\hline
\end{tabular}

Note: Dash (-) indicates that the test was not performed on the sample in question.

Two weeks later, a re-accumulated pleural effusion led to the admission we report (Figure 1). Our patient underwent a video-assisted thoracoscopic surgical biopsy. The pleural fluid again demonstrated a lymphocytosis with benign cytology and an adenosine deaminase assay yielded a value of 23.8 units per liter (Table 1). Multiple pleural biopsies demonstrated inflammation without any well-formed granulomata. All cultures of pleural fluid and biopsies were TB negative. Our patient's pleural fluid, which had not been tested serologically on previous thorocenteses, manifested a low complement with a total complement activity $(\mathrm{CH} 50)$ of 10, but was antinuclear antibody (ANA) negative. The serum ANA titer, however, was elevated to 1:160 with a speckled pattern, while a serum antihistone assay was negative. The isoniazid was discontinued, although rifampin and ethambutol were continued to complete a nine-month course as recommended by the infectious disease subspecialty consultants. There have been no further pleural effusions over two years of follow-up.

\section{Discussion}

The application of immunological rebound as an explanation for this case, in which the anti-TB therapy was empiric and without a convincingly demonstrated infection, is not consistent with the specific clinical data in hand. Moreover, the adenosine deaminase value we observed was below the cut-off point $(<35 \mathrm{U} / \mathrm{L})$ associated with a greater than $90 \%$ negative predictive value for pleural TB, even if the pre-test likelihood of disease was as high as 70\% [5]. A close examination of similar cases indicates that new effusions can follow the initiation of isoniazid therapy and resolve once that drug is discontinued [2,3]. Given the well-established association of isoniazid with drug-induced lupus (DIL), a lupus-related explanation of therapy-associated onset of pleural effusion in TB is quite plausible and avoids the presumed paradox of immunological rebound. Of note, serositis is a common feature of DIL, with pleural effusions occurring in as many as $50 \%$ of cases, depending on the inciting drug. DIL serologies were documented in three previous cases of pleural effusions after anti-TB therapy involving isoniazid [6,7]. These cases showed a markedly decreased $\mathrm{CH} 50$ in pleural fluid and an elevated ANA in both the serum and the pleural fluid. Pericardial effusions resulting from isoniazid-induced lupus have also been reported [8]. The non-specific ANA serum elevation we observed is consistent with DIL, as was the pleural effusion resolution following the removal of isoniazid from the treatment regimen. Although we did not observe positive serum antihistone antibodies, these can be negative in as many as a quarter of DIL cases [9]. While DIL is occasionally associated with rifampin, the resolution of symptoms in the case despite continued exposure to this agent argues that it did not play a role [10].

\section{Conclusion}

The case we describe, in conjunction with other cases in the medical literature, underscores the likely pivotal role for DIL in at least some new or worsening effusions during isoniazid treatment. Isoniazid-induced lupus should be considered in the differential diagnosis of a treatment emergent effusion and appropriate diagnostic tests should be pursued before such an effusion is attributed to a paradoxical pleural response.

\section{Consent}

Written informed consent was obtained from the patient for publication of this case report and any accompanying images. A copy of the written consent is available for review by the Editor-in-Chief of this journal.

\section{Abbreviations}

ANA: anti-nuclear antibody; DIL: drug-induced lupus; RIPE: rifampin, isoniazid, pyrazinamide, ethambutol; TB: Mycobacterium tuberculosis infection.

\section{Acknowledgements}

We would like to thank Dr Kirk Jones for reviewing the pathology specimens with us, and Dr Daniel Pound and the patient's daughter for keeping us updated on the patient's clinical progress following discharge. 


\section{Authors' contributions}

Both W and PB were directly involved in the care of the patient during the admission described in this report. W was a major contributor in writing the manuscript with significant overall guidance and editorial assistance from PB. Both authors read and approved the final manuscript.

\section{Competing interests}

The authors declare that they have no competing interests.

Received: 6 June 2011 Accepted: 30 November 2011

Published: 30 November 2011

\section{References}

1. Ferrer J: Pleural tuberculosis. Eur Respir J 1997, 10:942-947.

2. Singh SK, Ahmad Z, Pandey DK, Gupta V, Naaz S: Isoniazid causing pleural effusion. Indian J Pharmacol 2008, 40(2):87-88.

3. Gupta RC, Dixit R, Purohit SD, Saxena A: Development of pleural effusion in patients during anti-tuberculous chemotherapy: analysis of twentynine cases with review of literature. Indian J Chest Dis Allied Sci 2000, 42:161-166.

4. Al-Majed SA: Study of paradoxical response to chemotherapy in tuberculous pleural effusion. Respir Med 1996, 90:211-214.

5. Porcel JM, Esquerda A, Bielsa S: Diagnostic performance of adenosine deaminase activity in pleural fluid: a single-center experience with over 2100 consecutive patients. Eur J Int Med 2010, 21:419-423.

6. Hiraoka K, Nagata N, Kawajiri T, Suzuki K, Kurokawa S, Kido M, Sakamoto N: Paradoxical pleural response to antituberculous chemotherapy and isoniazid-induced lupus. Review and report of two cases. Respiration 1998, 65:152-155.

7. Jouneau S, Volatron AC, Brinchault G, Morel V, Belleguic C, Delaval P: Pleuro-péricardite induite par l'isoniazide [Isoniazid-induced pleuropericarditis]. Rev Pneumol Clin 2003, 59(6):357-359.

8. Siddiqui MA, Khan IA: Isoniazid-induced lupus erythematosus presenting with cardiac tamponade. Am J Therapurt 2002, 9:163-165.

9. Katz U, Zandman-Goddard G: Drug-induced lupus: an update. Autoimmun Rev 2010, 10:46-50.

10. Patel DG, Anstey AV: Rifampin-induced lupus erythematosus. Clin Expir Derm 2001, 26:60-62.

doi:10.1186/1752-1947-5-558

Cite this article as: Varenika and Blanc: A patient on RIPE therapy presenting with recurrent isoniazid-associated pleural effusions: a case report. Journal of Medical Case Reports 2011 5:558.

\section{Submit your next manuscript to BioMed Central and take full advantage of:}

- Convenient online submission

- Thorough peer review

- No space constraints or color figure charges

- Immediate publication on acceptance

- Inclusion in PubMed, CAS, Scopus and Google Scholar

- Research which is freely available for redistribution

Submit your manuscript at www.biomedcentral.com/submit
Biomed Central 